\title{
Model-based Analysis for the Thermal Management of Open-Cathode Proton Exchange Membrane Fuel Cell Systems concerning Efficiency and Stability
}

\author{
Stephan Strahl ${ }^{\mathrm{a}}$, Ramon Costa-Castellób,* \\ ${ }^{a}$ Institut de Robòtica i Informàtica Industrial (CSIC-UPC), Parc Tecnològic de \\ Barcelona, C/Llorens i Artigas 4-6, 08028 Barcelona, Spain \\ ${ }^{b}$ Universitat Politècnica de Catalunya (UPC); U Building (FME) ; Office : 507; C/ Pau \\ Gargallo, 5; 08028-Barcelona, Catalunya, Spain
}

\begin{abstract}
In this work we present a dynamic, control-oriented, concentrated parameter model of an open-cathode Proton Exchange Membrane fuel cell system for the study of stability and efficiency improvement with respect to thermal management. The system model consists of two dynamic states which are the fuel cell temperature and the liquid water saturation in the cathode catalyst layer. The control action of the system is the inlet air velocity of the cathode air flow manifold, set by the cooling fan, and the system output is the stack voltage. From the model we derive the equilibrium points and eigenvalues within a set of operating conditions and subsequently discuss stability and the possibility of efficiency improvement. The model confirms the existence of a temperature-dependent maximum power in the moderate temperature region. The stability analysis shows that the maximum power line decomposes the phase plane in two parts, namely stable and unstable
\end{abstract}

\footnotetext{
*Corresponding author
} 
equilibrium points. The model is capable of predicting the temperature of a stable steady-state voltage maximum and the simulation results serve for the design of optimal thermal management strategies.

Keywords: Open-cathode PEM fuel cell, Modeling, Stability analysis, Efficiency improvement

\section{Introduction}

Over the last decades great advancement in terms of materials, component design, production and system power density of Proton Exchange Membrane (PEM) fuel cells has been achieved. However, operating strategies for improving performance and durability by manipulation of operating conditions, such as temperature, humidity and reactant flow rates, have still not been sufficiently explored. One reason is the complex interconnection of performance, efficiency and durability of PEM fuel cells, which demands for an in-depth understanding of the competing effects when it comes to the design of proper control strategies.

In order to provide this deep understanding, experimental work is needed as well as a mathematical model that describes the involved physical phenomena. Mathematical modeling is an important tool in the development of fuel cells and control strategies, since the interplay of the involved phenomena is not always observable experimentally. Many detailed Computational Fluid Dynamics (CFD) models [1, 2, 3, 4, 5] have be developed and published over the last decade, that allow for numerical simulation of the detailed interactions between flow structure geometry, fluid dynamics, heat transfer and the electrochemical reactions, taking advantage 
of the steadily improving computational power. These modelling techniques provide insight into cell-internal spatially-resolved water distribution and its effect on fuel cell performance and durability. Furthermore they can support fuel cell designers in optimizing fuel cells before ever testing them in the laboratory.

Although most physical phenomena occurring in a PEM fuel cell can be incorporated in the macroscopic CFD models, it leads to time-consuming simulations with high computational costs, which makes them inconvenient for model-based controller development. A famous example of a dynamic fuel cell system model specially developed for control engineering was presented by [6]. The model describes the transient behaviour of the air compressor, the manifold filling dynamics, the reactant partial pressures and the membrane humidity. Stack voltage is calculated as a function of stack current, cathode pressure, reactant partial pressures, fuel cell temperature and membrane water content. However, the model neglects the electrochemical reaction kinetics and their dependence on the operating conditions. Moreover, stack temperature is treated as a constant parameter due to its slow time constant. Thus, system stability and robustness with respect to manipulation of the operating conditions, such as dynamic temperature changes, cannot be predicted.

Optimal water management in PEM fuel cells for optimized performance, always has to consider thermal management, as the fuel cell temperature has a strong effect on the water content in the ionomer of the membrane and the Catalyst Layers (CL): Water diffusion through the membrane as well as the water uptake dynamics of the ionomer are strong functions of 
temperature as shown by the experiments of [5] and [7], respectively. The temperature effects on water transport in the CLs are based on absorption and desorption of water into and from the ionomer, as well as evaporation and condensation in the pores of the CL. Water content in the catalyst layer has a direct effect on the electrochemical active surface area as analyzed by steady-state modeling of electrode structure effects on performance of [8] and [9]. If water is evacuated from the pores of the CL, the protons may not reach the active sites for the electrochemical reaction with the reactant gases and the electrons, which is the key factor of PEM fuel cell performance. This effect has also been shown experimentally by [10], explaining that the higher water evaporation flux at elevated temperature causes a reduction in active platinum sites. Even though many steady- state agglomerate models have shown up in the literature, a complete dynamic analysis of temperaturedependent water transport, including pore-filling dynamics, in relation with the electrode structure and its effects on performance is still not available, especially if embedded in a closed-loop fuel cell system model.

Optimal thermal management related to humidification is a crucial issue in every PEM fuel cell-based system. In this context, the use of external reactant gas humidification is intended to be avoided because the required humidifiers consume space and power, which decreases the overall system efficiency. [11] experimentally demonstrated the strong effects of low humidity operation on fuel cell performance. However, there is still a lack of information on how to control the temperature of a fuel cell system that works without reactant humidification with respect to performance optimization. The important links between temperature management and 
fundamental understanding of the catalyst layer humidification and activity for low humidity operation have not sufficiently been explored. As a first attempt to elucidate this problem, the work of [12] combines experimental analysis and theoretical studies of temperature effects on the performance of open-cathode PEM fuel cell systems for the design of optimal control strategies. The experimental analysis shows the great potential of improving the system performance by proper thermal management over the entire operation range. Especially at the higher current densities a significant system performance gain can be obtained due to improved reaction kinetics at higher temperatures. Dynamic, control-oriented models for fuel cell temperature, liquid water transport and the related electrochemistry have been developed and validated against the experiment. The authors concluded that performance improvement by temperature control means optimizing the tradeoff between increasing reaction kinetics and decreasing liquid water content in the catalyst layer.

In this work we extend and adapt the model of [12], described in section two, in order to perform analyses of the system's equilibrium points at different perturbations. Based on these analyses we discuss the system stability, dynamics and efficiency, shown in section three. Finally, applying the knowledge gained from the analysis, a temperature controller is designed in section four. 


\section{Model Description}

\subsection{Model discussion}

The modeled system in this work is the commercially available $100 \mathrm{~W}, 20$ cell PEM fuel cell system H-100 from Horizon Fuel Cells Technologies.This compact open-cathode system with an active area of $22.5 \mathrm{~cm}^{2}$ per cell, includes a single fan directly attached to the fuel cell housing, which removes heat from the stack by forced convection and at the same time provides oxygen to the cathode. Hence, the cathode reactant flow channels are as well used for the stack cooling. The cooling therefore depends on the ambient air temperature and the fan velocity. The fan power consumption directly affects the net power of the fuel cell system. The pressure-regulated anode inlet is supplied with dry hydrogen and the outlet features a normally-closed electromagnetic valve for dead-ended operation and purging.

For the model-based analysis in this paper we use a previously-developed model by the authors, presented in [12]. This baseline model consists of two dynamic states which are the fuel cell temperature, $\mathrm{T}_{\mathrm{fc}}$, and the liquid water saturation in the cathode catalyst layer, s. The liquid water saturation is defined as the ratio of the liquid volume to the total volume of void space in the porous structure [13]. The load current, I, and the cathode inlet air temperature, $\mathrm{T}_{\mathrm{amb}}$, can be considered as external disturbances. The control action of the system is the inlet air velocity $v_{\text {air }}$ of the cathode air flow manifold, set by the cooling fan. A change to the baseline model of [12] is that we define the output of the system as the fuel cell stack voltage $V_{\text {stack }}$ instead of the activation overpotential. The input variable, $v_{a i r}$, and the

disturbance, I, can be measured or set. The output variable, $V_{\text {stack}}$, the first 
state variable, $\mathrm{T}_{\mathrm{fc}}$, and the disturbance, $\mathrm{T}_{\mathrm{amb}}$, can be measured. The only variable which can not be measured directly is the second state variable, s. The state space representation of the model results in:

$$
\begin{aligned}
\dot{T}_{f c} & =K_{1} \cdot I-K_{1}^{\prime} \cdot I \cdot \mathrm{V}_{\text {stack }}+\left(K_{2} \cdot T_{a m b}-K_{2} \cdot T_{f c}\right) v_{a i r} \\
\dot{s} & =K_{3} \cdot I-K_{4} \cdot f_{p}\left(T_{f c}\right) \cdot s-f_{d}(s) \\
V_{\text {stack }} & =K_{7}-K_{6} \cdot I-K_{5} \cdot T_{f c} \cdot f_{a}\left(T_{f c}, s, I\right)
\end{aligned}
$$

The parameters $\left(K_{1}\right)$ to $\left(K_{7}\right)$, the functions $\left(f_{p}\right)$ and $\left(f_{d}\right)$ and the parameter values are defined in Appendix A together with the governing physical equations. The function $\left(f_{a}\right)$ describes a concentration-dependent Tafel approach for the cathode overpotential:

$$
f_{a}\left(T_{f c}, s, I\right)=\ln \left(\frac{I}{A_{g e o} i_{0}{ }^{A E C D}}\right)
$$

with

$$
i_{0}{ }^{A E C D}=i_{0}{ }^{r e f} \cdot\left(\frac{p_{O_{2}}}{P^{r e f}}\right)^{0.5} \cdot E C S A(s) \cdot \exp \left[\frac{-\Delta G^{*}}{R T}\left(1-\frac{T_{f c}}{T^{r e f}}\right)\right]
$$

The apparent exchange current density $\mathrm{i}_{0}{ }^{\mathrm{AECD}}$ combines the intrinsic catalytic activity of the $\mathrm{Pt}$ catalyst at reference conditions $\left(\mathrm{T}^{\mathrm{ref}}\right.$ and $\mathrm{P}^{\mathrm{ref}}$ ) $\mathrm{i}_{0}{ }^{\text {ref }}$ with the electrochemical active surface area (ECSA) and the dynamic states $\mathrm{T}_{\mathrm{fc}}$ and $\mathrm{s}[11,14,12]$. The change of the partial pressure of oxygen at the catalyst layer is assumed to be negligible due to operation at high cathode stoichiometries $(>20) . \Delta \mathrm{G}^{*}$ is the activation energy associated to the oxygen reduction reaction (ORR). The relationship between activation energy and the exchange current density follows an Arrhenius-type approach $[10,14]$. 
As shown in Eq.(5), the electrochemical active surface area (ECSA) is a function of the liquid water saturation. The relation between the ECSA and the liquid water saturation has been updated compared to the baseline model of [12] in order to include novel research results of the authors on the pore structure of the CL. Hence, the dynamic change of the active platinum surface area in the porous structure of the cathode CL is described by a similar approach as presented in [15] for a single pore size:

$$
\operatorname{ECSA}(s)=A^{o p t} \cdot\left(1-\frac{s^{o p t}-s}{s^{o p t}}\right)^{\frac{1}{3}}
$$

where $\mathrm{A}^{\mathrm{opt}}$ is the optimally achievable ECSA at the optimal liquid water saturation $\mathrm{s}^{\mathrm{opt}}$ under the systems' restricted operating conditions. Accordingly, at $\mathrm{s}^{\mathrm{opt}}$ the entire Pt surface area is available for the electrochemical reaction, which can be estimated by the product of Pt loading and the specific catalyst area [14], also known as electrode roughness factor. This closes the equation system of the model. More details on the physical background of the baseline model and parameter tuning are available in [12]. In the following sections the updated baseline model is used to perform novel analyses of the system's equilibrium points.

\subsection{Control action dicussion}

In order to simplify equations (1)-(3), the following variable change is applied:

$$
v_{a i r} \triangleq \frac{v}{K_{2} T_{a m b}-K_{2} T_{f c}}
$$


where $v$ corresponds to the extracted heat in $\mathrm{J} / \mathrm{s}$. With this variable change the equations result in:

$$
\begin{aligned}
\dot{T}_{f c} & =K_{1} \cdot I-K_{1}^{\prime} \cdot I \cdot \mathrm{V}_{\text {stack }}+v \\
\dot{s} & =K_{3} \cdot I-K_{4} \cdot f_{p}\left(T_{f c}\right) \cdot s-f_{d}(s) \\
V_{\text {stack }} & =K_{7}-K_{6} \cdot I-K_{5} \cdot T_{f c} \cdot f_{a}\left(T_{f c}, s, I\right)
\end{aligned}
$$

Note that these equations do not depend on $T_{a m b}$, which simplifies Eq. (8). The variable change (7) is well defined when $K_{2}\left(T_{a m b}-T_{f c}\right) \neq 0$. As $K_{2}$ is a constant different from zero, only at $T_{a m b}=T_{f c}$ the denominator is zero. Whenever current is drawn from the fuel cell, heat is generated due to the exothermic nature of the electrochemical reaction. Thus, at a constant ambient temperature and a started-up fuel cell, the fuel cell temperature is always greater than the ambient temperature, resulting in a non-zero denominator for Eq. (7). This makes Eq. (7) well defined in a realistic scenario.

The new control variable $v$ maintains a physical meaning concerning the thermal system, since it represents the heat extracted from the fuel cell. This may be even closer connected to temperature changes than the air velocity. From the practical point of view using Eq. (7) has a drawback, which is the need to measure the ambient temperature. Fortunately, nowadays there exist many ways to measure this variable at a low cost. 


\section{Model analysis}

\subsection{Equilibrium points}

\subsubsection{Definition}

In dynamic system analysis, equilibrium points play a key role [16]. These points define configurations in which the system can be considered stationary; consequently the derivatives of the state variables have to be zero. For the system under study the equilibrium points correspond to: ${ }^{1}$

$$
\begin{aligned}
0 & =K_{1} \cdot I^{*}-K_{1}^{\prime} \cdot I^{*} \cdot V_{\text {stack }}^{*}+v^{*} \\
0 & =K_{3} \cdot I^{*}-K_{4} \cdot f_{p}\left(T_{f c}^{*}\right) \cdot s^{*}-f_{d}\left(s^{*}\right) \\
V_{\text {stack }}^{*} & =K_{7}-K_{6} \cdot I^{*}-K_{5} \cdot T_{f c}^{*} \cdot f_{a}\left(T_{f c}^{*}, s^{*}, I^{*}\right) .
\end{aligned}
$$

\subsubsection{Computing equilibrium points}

The equations (11)-(13) are highly nonlinear, which makes it difficult to analyze them analytically. From the implicit function theorem [16] it is possible, at least locally, to write :

$$
\begin{aligned}
T_{f c}^{*} & =\phi_{1}\left(I^{*}, v^{*}\right) \\
s^{*} & =\phi_{2}\left(I^{*}, v^{*}\right) \\
V_{\text {stack }}^{*} & =\phi_{3}\left(I^{*}, v^{*}\right)
\end{aligned}
$$

The expressions $\phi_{1}, \phi_{2}$ and $\phi_{3}$ were not determined analytically, hence the equilibrium points have been characterized numerically. A direct approach would be fixing $I^{*}$ and $v^{*}$ and numerically solving (11)-(12) for $T_{f c}^{*}$ and $s^{*}$. As (11)-(12) are highly nonlinear equations numerical methods require good

\footnotetext{
$1 *$ is used to note that the variable fulfills equilibrium point conditions
} 
initial conditions to achieve convergence. Obtaining a good set of initial conditions by guessing $T_{f c}^{*}$ and $s^{*}$ approximately is not trivial.

In order to simplify this procedure, the analytic expressions of Eqs. (11)(12) have been studied. If Eq. (13) is inserted into Eq. (11), it is possible to isolate $s^{*}$ from the resulting equation. Consequently it is possible to obtain an analytical expression for $s^{*}$ :

$$
s^{*}=\phi_{s}\left(T_{f c}^{*}, I^{*}, v^{*}\right)
$$

This expression is introduced into Eq. (12) :

$$
0=K_{3} \cdot I^{*}-K_{4} \cdot f_{p}\left(T_{f c}^{*}\right) \cdot \phi_{s}\left(T_{f c}^{*}, I^{*}, v^{*}\right)-f_{d}\left(\phi_{s}\left(T_{f c}^{*}, I^{*}, v^{*}\right)\right)
$$

The resulting equation (18), although highly nonlinear, is a scalar function with one unknown, $v^{*}$, and thus easier to handle. In order to analyze the equilibrium points, a grid is defined for $\left(T_{f c}^{*}, I^{*}\right)$. Thus, equation (18) can be solved numerically by a zero finding algorithm. In our case, the equation is solved using a zero finding numerical algorithm, in particular the MATLAB function ' $f z e r o$ ' is used, which does not need to compute the function derivative. In order to achieve good precision, an absolute tolerance of $10^{-10}$ has been used.

Finally, $s^{*}$ is obtained using Eq. (17) and subsequently Eq. (13) can be solved for $V_{\text {stack }}^{*}$.

\subsubsection{Equilibrium points analysis}

Figure 1.a shows the obtained equilibrium points when fixing $I^{*}$ at different levels and solving for $T_{f c}^{*}$ and $v^{*}$. For each current a curve is obtained, which is plotted in color scale. This color scale corresponds to 
the respective fuel cell power, $V_{\text {stack }}^{*} I^{*}$, for each point. This analysis has been restricted to the following variables ranges: $I^{*} \in[2.5,6.5] \mathrm{A}, s^{*} \in[0.01,0.8]$, $T_{f c}^{*} \in[25,62]^{\circ} \mathrm{C}$ and $v_{\text {air }}^{*} \in[0.1,1] \mathrm{m} / \mathrm{s}$. The current range corresponds to a current density range of $i^{*} \in[0.11,0.29] \mathrm{A} / \mathrm{cm}^{2}$.

The specified set of operating conditions are considered as the relevant range of interest for each variable, respectively, of the studied open-cathode fuel cell stack. More details on operation conditions of open-cathode stack and their effect on performance can be found in previous works of the authors $[17,12]$.

Besides the equilibrium points, Figure 1.a shows a black curve that corresponds to the maximum power obtained for each constant current setpoint. One can see that there is a tradeoff between increasing the fuel cell temperature and decreasing the liquid water saturation in the cathode catalyst layer. On the one hand increasing the operating temperature improves the activity of the ORR, while on the other hand it increases evaporation of liquid water in the pores of the CL, which results in decreased proton conductivity and thus decreases the active platinum surface. Therefore, the points of maximum power can be found at moderate temperatures instead at the highest allowable temperatures. The described model thus helps to predict the optimal temperature setpoint.

Figure 1.b shows, in more detail, the same data for a current of $I^{*}=6.5 \mathrm{~A}$. Regarding the allowable temperature range, a performance improvement of about $1.7 \mathrm{~W}$ can be obtained at that current setpoint, which is about $2.2 \%$ of the maximum power. This increase in fuel cell power can be obtained by carefully adapting the cooling air flow, as experimentally shown in [12]. 
Figure 2 shows the evolution of $T_{f c}^{*}$ versus $v_{\text {air }}{ }^{*}$ for different current setpoints. Similarly to previous case, the black line corresponds to the points with maximum power. It can be observed that the target control range for the air velocity in order to achieve maximum fuel cell power is between 0.15 and $0.25 \mathrm{~m} / \mathrm{s}$, while the fan of this open-cathode system is dimensioned for velocities up to $1 \mathrm{~m} / \mathrm{s}$. Consequently, in order to reduce system cost and weight, and at the same time improve the controllability of the cathode air velocity, the fan could be sized down to a smaller operating range, at least for stationary operation. However, there will be a tradeoff in terms of the maximum cooling flow and the resulting dynamic temperature response to a change in the current setpoint. Thus, a two-fan solution may become attractive, especially considering the possible reduction in auxiliary power consumption of the cooling fan when operating at low velocities for maximum power during stationary operation. This leads to an improved fuel cell system net power.

\subsection{Stability analysis}

\subsubsection{Local stability}

The equilibrium points determined in section 3.1 may be stable or unstable [16]. In case of stable points it is possible to operate in openloop around them. For instance, if the system is at a specific point of operation $\left(T_{f c}, s\right)$ close to the equilibrium point $\left(T_{f c}^{*}, s^{*}\right)$, and the inputs, $(I, v)$, are maintained constant at the appropriate value $\left(I^{*}, v^{*}\right)$, respectively, the system dynamics will take the system to the equilibrium point $\left(T_{f c}^{*}, s^{*}\right)$. In the case of unstable points the system will tend to drift away from the equilibrium point, consequently it is not possible to operate in their vicinity 
in open-loop.

In order to determine if an equilibrium point $\left(T_{f c}^{*}, s^{*}\right)$ is stable or unstable the Lyapunov's linearization method is performed [18]. Firstly, equations (1)(3) are written as:

$$
\dot{\mathbf{x}}=\mathbf{f}(\mathbf{x}, \mathbf{u})
$$

where $\mathbf{x}=\left[T_{f c}, s\right]^{T}$ and $\mathbf{u}=[I, v]^{T}$. For equilibrium points applies:

$$
\mathbf{0}=\mathbf{f}\left(\mathbf{x}^{*}, \mathbf{u}^{*}\right)
$$

According to Lyapunov's approach [16] an equilibrium point $\mathbf{x}^{*}=\left[T_{f c}^{*}, s^{*}\right]^{T}$, $\mathbf{u}^{*}=\left[I^{*}, v^{*}\right]^{T}$ will be stable if the eigenvalues of $\left.\frac{\partial \mathbf{f}}{\partial \mathbf{x}}\right|_{\mathbf{x}^{*}, \mathbf{u}^{*}}$ are placed in the left half of the complex plane.

Figure 3 shows the system eigenvalues for the studied second-order system at different equilibrium points (the eigenvalues are drawn as a function of $\left.T_{f c}^{*}\right)$. Figure 3.a corresponds to the minimum eigenvalue while Figure 3.b corresponds to the maximum one. When the curves cross the red line in Figure 3.b, a transition from stability to instability occurs. The circles over the curves in Figure 3.a indicate the operation points where this transition occurs in the minimum eigenvalue. When one of the eigenvalues becomes positive the complete system becomes unstable, this induces a decomposition of the equilibrium point into stable and unstable ones. This decomposition is visualized in Figure 4. Stable equilibrium points are plotted in blue while unstable ones are plotted in red. As it can be seen, the maximum power line decomposes the plane $T_{f c}-s$ in two parts, namely stable and unstable equilibrium points. This coincides reasonably well with the observed instabilities in the voltage response to open-loop fan flow steps 
in the experiments of [12], where the stack voltage starts to drifts away after increasing the temperature above $45^{\circ} \mathrm{C}$ at a current of $4 \mathrm{~A}$.

The trajectories of Eq. (19) around the equilibrium point can be approximated by those of the following linear system:

$$
\dot{\Delta \mathbf{x}}=\left(\left.\frac{\partial \mathbf{f}}{\partial \mathbf{x}}\right|_{\mathbf{x}^{*}, \mathbf{u}^{*}} \cdot \Delta \mathbf{x}\right)+\left(\left.\frac{\partial \mathbf{f}}{\partial \mathbf{u}}\right|_{\mathbf{x}^{*}, \mathbf{u}^{*}} \cdot \Delta \mathbf{u}\right)
$$

where $\Delta \mathbf{x}=\mathbf{x}-\mathbf{x}^{*}$ and $\Delta \mathbf{u}=\mathbf{u}-\mathbf{u}^{*}$. In case that $\Delta \mathbf{u}=\mathbf{0}$, the trajectories are defined by :

$$
\Delta \mathbf{x}=\left.\frac{\partial \mathbf{f}}{\partial \mathbf{x}}\right|_{\mathbf{x}^{*}, \mathbf{u}^{*}} \cdot \Delta \mathbf{x}
$$

The shape of the trajectories of this system depend on the eigenvalues of $\left.\frac{\partial \mathbf{f}}{\partial \mathbf{x}}\right|_{\mathbf{x}^{*}, \mathbf{u}^{*}}$.

As shown above, both eigenvalues are real; the minimum eigenvalue is always negative (stable behavior), but the maximum eigenvalue is negative for small temperatures and moves into positive (unstable behavior) as the temperature increases. Under operating conditions where the maximum eigenvalue is positive the complete system is unstable. As shown in Fig. 3, for stable equilibrium points the minimum eigenvalues move approximately from $-3.5 \cdot 10^{-3}$ to $-1.54 \cdot 10^{-3}$ while the maximum lies between $-3.5 \cdot 10^{-4}$ and 0 .

Figure 5 shows the trajectories obtained in the plane $T_{f c}-s$ for different initial conditions around three different equilibrium points for a constant current of $I^{*}=6.5 \mathrm{~A}$ and three different values of $v^{*}$. Second order systems with two real eigenvalues evolve over the phase-plane converging to a line which is defined by the eigenvectors of $\left.\frac{\partial \mathbf{f}}{\partial \mathbf{x}}\right|_{\mathbf{x}^{*}, \mathbf{u}^{*}}$. In the studied fuel cell system this convergence line is almost aligned with the curve defined by 
maintaining $I^{*}$ constant and sweeping the value of $v^{*}$.

Additionally, it can be stated that the time required to converge to the line is about one order of magnitude faster ${ }^{2}$ than the required time to reach the equilibrium point once already over the line.

\subsubsection{Global stability}

In the previous section a local stability analysis has been performed, which provides information about the system behavior around the equilibrium points. In this section the stability analysis is completed with the formulation of a Lyapunov function [16], which in turn allows to extend the study to a wider region of the phase plane.

Although the analyzed model provides a good description of most relevant phenomena involved in the PEM fuel cell operation, it is not a purely theoretical physics model. Instead, the model combines physical laws, empirical equations and behavior inspired laws. This makes difficult to derive a Lyapunov function based on the energy of the system. Instead the information obtained in the previous section is used to formulate the function. The Lyapunov function must be positive definite in the region of interest and it must have a minimum at the equilibrium points. Due to these requirements the following Lyapunov function is proposed:

$$
V(\mathbf{x})=(\mathbf{x}-\boldsymbol{\phi}(\mathbf{u}))^{T} \mathbf{P}(\mathbf{x}-\boldsymbol{\phi}(\mathbf{u}))
$$

where $\mathbf{x}=\left[T_{f c}, s\right]^{T}, \mathbf{u}=[I, v]^{T}$ and $\mathbf{P}>0$. The function $\boldsymbol{\phi}=$ $\left[\phi_{1}(\mathbf{u}), \phi_{2}(\mathbf{u})\right]^{T}$ relates the external inputs with the state variables at the

\footnotetext{
${ }^{2}$ It depends mainly on the minimum eigenvalue and it is around $2 \cdot 10^{3} \mathrm{~s}$.
} 
equilibrium points (section 3.1). The Lyapunov function (22) is always positive and is equal to zero at the equilibrium points. In the following, the derivative of (22) over the system trajectories is analyzed, and it is shown that the derivative is always negative in the region of interest. Firstly, an appropriate value of $\mathbf{P}$ is obtained by solving the following LMI set:

$$
\begin{aligned}
\mathbf{P} & >0 \\
\mathbf{A}_{k}^{T} \mathbf{P}+\mathbf{P A}_{k} & <0, k=1, \cdots, n
\end{aligned}
$$

where $\mathbf{A}_{k}$ corresponds to the Jacobian matrix defined in (21) at different stable equilibrium points.

To solve the LMI a reduced number of points $(n=20)$, equidistributed over the region of interest, has been used. Finally, the obtained value for $\mathbf{P}$ is checked with an enlarged number of points $(n=10000)$ with a similar distribution. A value which fulfills (23) and (24) in all tested points is:

$$
\mathbf{P}=\left[\begin{array}{cc}
1 & 103.2622 \\
103.2622 & 56364.83646
\end{array}\right]
$$

The obtained derivative for $(22)$ is:

$\dot{V}(\mathbf{x})=(\mathbf{x}-\phi(\mathbf{u}))^{T} \mathbf{P}\left(\mathbf{f}(\mathbf{x}, \mathbf{u})-\frac{\partial \phi}{\partial \mathbf{u}} \dot{\mathbf{u}}\right)+\left(\mathbf{f}(\mathbf{x}, \mathbf{u})-\frac{\partial \phi}{\partial \mathbf{u}} \dot{\mathbf{u}}\right)^{T} \mathbf{P}(\mathbf{x}-\boldsymbol{\phi}(\mathbf{u}))(26)$ where $\mathbf{f}$ has been defined in (19). When $\mathbf{x}$ is close to an equilibrium point $\mathbf{x}^{*}=\boldsymbol{\phi}\left(\mathbf{u}^{*}\right)$ and $\mathbf{u}=\mathbf{u}^{*}$, the previous equation can be approximated by :

$$
\dot{V}(\mathbf{x}) \approx\left(\mathbf{x}-\phi\left(\mathbf{u}^{*}\right)\right)^{T}\left(\left.\mathbf{P} \frac{\partial \mathbf{f}}{\partial \mathbf{x}}\right|_{\phi\left(\mathbf{u}^{*}\right), \mathbf{u}^{*}}+\left.\frac{\partial \mathbf{f}}{\partial \mathbf{x}}\right|_{\phi\left(\mathbf{u}^{*}\right), \mathbf{u}^{*}} ^{T} \mathbf{P}\right)\left(\mathbf{x}-\boldsymbol{\phi}\left(\mathbf{u}^{*}\right)\right)
$$

which is negative definite for the stable equilibrium points by construction. 
The complexity of the equations involved in $\mathbf{f}$ makes difficult to analytically prove that (26) is negative definite. However an extensive numerical analysis has been performed within the region of interest. This study concludes that (26) is negative definite when $\mathbf{u}^{*}$ is constant and $\mathbf{x}^{*}=\boldsymbol{\phi}\left(\mathbf{u}^{*}\right)$ and $\mathbf{u}^{*}$ define a stable equilibrium point. To illustrate this, Figure 6 shows the evolution of $\dot{V}$ for four different values of $\mathbf{u}^{*}$. In each subfigure in Figure 6 the studied equilibrium point is shown, as well as the boundary between stable and unstable equilibrium points and the evolution of $\dot{V}$.

As depicted in Figure $6, \dot{V}$ is negative definite in the region of interest. As previously shown in the local analysis there is a direction in which the convergence range is much faster than in the complementary one. This study proves that, within the region of interest, the equilibrium points are globally asymptotically stable. Even if the system begins with initial conditions which are in the region of unstable equilibrium points the system will converge to the equilibrium point.

\subsection{Efficiency improvement evaluation}

As explained in the introduction, proper temperature control of the studied open-cathode system can lead to power and efficiency improvement. Figure 7 shows the stack voltage as a function of temperature at several constant power setpoints. As shown experimentally in our previous publication [12], the simulation results in Figure 7 depict that a performance improvement for any load request can be obtained by temperature control. Secondly, a clear maximum of the possible performance gain can be observed, with subsequent power loss after passing the optimal temperature.

Figure 8 shows the performance and efficiency gain for a load request of 
$70 \mathrm{~W}$ in more detail. Between 35 and $60^{\circ} \mathrm{C}$ the possible relative performance improvement of the fuel cell stack (defined by the stack voltage gain at constant power here) is $1.6 \%$, considering open-loop manipulation of the cooling fan flow. This is further increased to about 3.5\% for the fuel cell system, since the voltage increase is obtained via a reduction in the cooling flow, which decreases the parasitic power consumption of the fan [12].

Consequently, the stack current at constant power in the same temperature range drops by about $0.1 \mathrm{~A}$. Following Faraday's law, this decreases the system's fuel consumption at $70 \mathrm{~W}$ by $1.6 \%$. This shows how proper thermal management provides an attractive option to increase the efficiency of the open-cathode system just by software, without modifying any system component. Additionally, in combination with the described down-sizing of the cooling fan, as discussed in section 3.1.3, the efficiency may be increased even further.

\section{Control Scheme}

As discussed in the previous section, adequate temperature control can increase the system efficiency. For this purpose, a temperature controller, based on the presented model, is proposed. Although in section 3.2.2 a Lyapunov function has been formulated, a controller based on that would be complex. Hence, a simpler controller based on the system analysis and comprehension is proposed. This controller is composed by two parts, a feedforward term, which allows to achieve the desired equilibrium point and a feedback controller, which handles the plant uncertainty and imposes a fast closed-loop behavior. Figure 9 contains a complete scheme of the closed-loop 
system.

In the equilibrium point analysis of section 3.1 a relationship, between $T_{f c}^{*}$, $v^{*}$ and $I^{*}$ has been developed $\left(T_{f c}^{*}=\phi_{1}\left(I^{*}, v^{*}\right)\right)$. In order to take advantage of this expression for the controller design, the relationship is transformed into the following:

$$
v^{*}=\gamma\left(T_{f c}^{*}, I^{*}\right)
$$

which provides the required control action to take the system to the equilibrium point defined by $T_{f c}^{*}$ and $I^{*}$. According to the stability analysis, in case of a perfect system knowledge this control action would be sufficient to take system to the equilibrium point (the stable equilibrium points have been proven to be globally asymptotic stable). As discussed in section 3.1, it is not possible to obtain an analytic expression for $\phi_{1}$, the same applies for $\gamma$. Therefore, a polynomial has been interpolated through numerical fitting instead:

$$
v^{*}=\gamma\left(T_{f c}^{*}, I^{*}\right)=\beta_{00}+\beta_{10} T_{f c}^{*}+\beta_{01} I^{*}+\beta_{11} T_{f c}^{*} I^{*}+\beta_{02}\left(I^{*}\right)^{2}
$$

where $\beta_{00}=0.0326, \beta_{10}=-9.593 \cdot 10^{-5}, \beta_{01}=-0.06164, \beta_{11}=0.0001137$, $\beta_{02}=-0.0009065$.

In order to design a linear feedback controller, the linearized model described in section 3.2 will be used. As previously discussed, locally the system behaves like a second order system with two real poles and one zero. Taking $\Delta v_{a i r}$ as input and $\Delta T_{f c}$ as output, the transfer function has the following shape:

$$
G(s)=\frac{s+z}{(s+p 1)(s+p 2)}
$$

with $z \in[0.00134,0.00384], p_{1} \in[0.0017,0.00417]$ and $p_{2} \in\left[5.34 \cdot 10^{-5}, 0.00105\right]$. 
These intervals have been derived from analyzing the linearized model in a region of interest defined by $T_{f c}^{*}=[301.12,332.98] \mathrm{K}, s^{*}=[0.0638,0.16129]$ and $I^{*}=[3,9]$ A, which includes almost all the relevant operation points.

Figure 10 shows the Bode plot of some plants belonging to this transfer function family of Eq. (30). Analyzing the frequency responses and using conventional loop shaping methods, the following PI controller is proposed:

$$
P I(s)=1+\frac{1}{10 \cdot s}=\frac{s+\frac{1}{10}}{s} \text {. }
$$

This controller guarantees robust stability ( $\mu$-analysis methods have been applied) and offers an excellent performance both in the time and the frequency domain. Figure 11 shows the step response for the closed-loop system (modified sensitivity function) for some members of the transfer function family and the proposed PI controller. All step responses in Figure 11.a are very similar and the settling time is around 30s in all cases. Figure 11.b shows the control action generated by the proposed PI controller.

Figure 12 shows the magnitude of the sensitivity function frequency response for some members of the family and the proposed PI controller. All systems show a very small error within the working bandwidth (less than $-40 \mathrm{~dB}$ for frequencies lower than $10^{-2} \mathrm{rad} / \mathrm{s}$ ), no noise amplification at high frequency and an excellent robustness (all family members have a distance from the Nyquist plot to -1 point greater or equal to 1 ).

The controller design is completed with a simple anti-windup scheme, $K_{a w}$, which helps to handle control action saturation when big changes in the reference are applied. Finally, Figure 13 shows the evolution of the complete system (nonlinear model) when following a given trajectory for the temperature reference. In this simulation two different constant current 
values are set $I=4 \mathrm{~A}$ and $I=6 \mathrm{~A}$, respectively. The controller is performing correctly and smoothly, even for large changes in the temperature reference. The upper and lower saturation levels of the cooling fan, as experimentally characterized in a previous work [12], are properly managed by the antiwindup scheme. The heating dynamic of the fuel cell after an increase in the temperature setpoint depends on the generated heat (setpoint reached faster at higher currents). Accordingly, in order to reach the setpoint as fast as possible, the controller directly sets the minimum air flow. Vice versa, a decrease in the temperature setpoint causes the fan to reach the upper air

flow limit (full power). However, as already explained in section 3.1.3, the control action plot in Figure 13 shows that the fan is over-dimensioned.

\section{Conclusions}

In the presented work we expanded our earlier published control-oriented model of an open-cathode PEM fuel cell system [12] and used it for the study of the system equilibrium points, their dynamics and stability. Among the set of equilibrium points, the points of maximum power as a function of temperature and liquid water saturation in the cathode catalyst layer were characterized. A tradeoff between increasing the fuel cell temperature and decreasing the liquid water saturation in the cathode catalyst layer was visualized. Hence, the operating points of maximum power can be found at moderate temperatures instead at the highest allowable temperatures.

The stability analysis shows that the connection of points with maximum power decomposes the phase plane in two parts, namely stable and unstable equilibrium points. Moreover, the maximum eigenvalues are approximately 
10 times smaller (in magnitude) than the minimum eigenvalues. The eigenvalue study also revealed a settling time of around $2 \cdot 10^{4} \mathrm{~s}$. This very slow dynamic is due to the slow process of water sorption and desorption into and from the membrane. Nevertheless, the 10 times faster maximum eigenvalue brings the system relatively fast close to the final equilibrium point.

The developed model-based temperature controller has been validated in simulation with the complete nonlinear model. This controller is quite simple but assures robust stability and excellent tracking performance throughout the operation range, which is the base for further fuel cell system control objectives, such as overall system efficiency optimization.

In conclusion, the model is capable of predicting the temperature of a stable steady-state voltage maximum and the simulation results in this work serve for the design of optimal thermal management strategies. Efficient temperature control may improve the net efficiency of the studied system by around $3.5 \%$, as shown by the simulation results. Besides efficiency improvements, a well-designed controller may also improve the characterized slow open-loop time response of the system.

\section{Acknowledgements}

This work is partially funded by the national project MICINN DPI2015-

69286-C3-2-R MINECO/FEDER and 2014 SGR 267 of the AGAUR agency of the Generalitat de Catalunya. 


\section{References}

[1] H. Ju, H. Meng, C. Wang, A single-phase, non-isothermal model for PEM fuel cells, International Journal of Heat and Mass Transfer 48 (7) (2005) 1303-1315. doi:10.1016/j.ijheatmasstransfer.2004.10.004.

URL http://linkinghub.elsevier.com/retrieve/pii/S0017931004004685

[2] S. Basu, J. Li, C.-Y. Wang, Two-phase flow and maldistribution in gas channels of a polymer electrolyte fuel cell, Journal of Power Sources 187 (2) (2009) 431-443. doi:10.1016/j.jpowsour.2008.11.039.

URL http://linkinghub.elsevier.com/retrieve/pii/S0378775308021642

[3] Y. Wang, C.-Y. Wang, A Nonisothermal, Two-Phase Model for Polymer Electrolyte Fuel Cells, Journal of The Electrochemical Society 153 (6) (2006) A1193-A1200. doi:10.1149/1.2193403.

URL http://link. aip.org/link/JESOAN/v153/i6/pA1193/s1\&Agg=doi

[4] Y. Wang, C.-Y. Wang, Two-Phase Transients of Polymer Electrolyte Fuel Cells, Journal of The Electrochemical Society 154 (7) (2007) B636-B643. doi:10.1149/1.2734076.

URL http://link. aip.org/link/JESOAN/v154/i7/pB636/s1\&Agg=doi

[5] S. Strahl, A. Husar, M. Serra, Development and experimental validation of a dynamic thermal and water distribution model of an open cathode proton exchange membrane fuel cell, Journal of Power Sources 196 (9) (2011) 4251-4263. doi:10.1016/j.jpowsour.2010.10.074.

[6] J. T. Pukrushpan, A. G. Stefanopoulou, H. Peng, Control of fuel cell 
power systems: principles, modeling, analysis, and feedback design, Springer, 2004.

[7] P. W. Majsztrik, M. B. Satterfield, A. B. Bocarsly, J. B. Benziger, Water sorption, desorption and transport in nafion membranes, Journal of Membrane Science 301 (1) (2007) 93-106.

[8] Q. Wang, M. Eikerling, D. Song, Z. Liu, Structure and performance of different types of agglomerates in cathode catalyst layers of PEM fuel cells, Journal of Electroanalytical Chemistry 573 (1) (2004) 61-69.

[9] M. Eikerling, Water management in cathode catalyst layers of PEM fuel cells a structure-based model, Journal of The Electrochemical Society 153 (3) (2006) E58-E70.

[10] C. Song, Y. Tang, J. L. Zhang, J. Zhang, H. Wang, J. Shen, S. McDermid, J. Li, P. Kozak, PEM fuel cell reaction kinetics in the temperature range of 23-120 c, Electrochimica Acta 52 (7) (2007) 2552-2561.

[11] J. Zhang, Y. Tang, C. Song, X. Cheng, J. Zhang, H. Wang, PEM fuel cells operated at $0 \%$ relative humidity in the temperature range of $23-$ 120 c, Electrochimica Acta 52 (15) (2007) 5095-5101.

[12] S. Strahl, A. Husar, P. Puleston, J. Riera, Performance improvement by temperature control of an open-cathode PEM fuel cell system, Fuel Cells 14 (2014) 466-478.

[13] U. Pasaogullari, C. Wang, Liquid water transport in gas diffusion layer of polymer electrolyte fuel cells, Journal of the Electrochemical Society 151 (3) (2004) A399-A406. 
[14] F. Barbir, PEM Fuel cells: Theory and Practice, Elsevier Academic Press, 2005.

[15] S. Strahl, A. Franco, A. Husar, Electrode structure effects on the performance of open-cathode proton exchange membrane fuel cells: a multiscale modeling approach, International Journal of Hydrogen Energy 39 (2014) 9752-9767.

[16] H. K. Khalil, Nonlinear Systems, 3rd Edition, Prentice Hall, Upper Saddle River, New Jersey, 2002.

[17] S. Strahl, A. Husar, J. Riera, Experimental study of hydrogen purge effects on performance and efficiency of an open-cathode PEM fuel cell system, Journal of Power Sources 248 (2014) 474-482.

[18] J.-J. E. Slotine, W. Li, Applied Nonlinear Control, Prentice-Hall Inc., 1991, iSBN 0-13-040890-5.

\section{Appendix A. Model details}

The entire model has been described and experimentally validated in an earlier publication of the authors [12]. All parameter values can be found in [12]. In the following, the governing equations are presented in short, together with the derived parameters for the state-space representation.

Appendix A.1. Governing equations

The governing equations for the system model are the heat transport equation, A.1, and the liquid water transport equation, A.4, for the fuel cell cathode catalyst layer. 
Heat transport:

$$
\frac{d T_{f c}}{d t}=\frac{1}{m_{f c} C_{p, f c}}\left(\dot{Q}_{t o t}-\dot{Q}_{c o o l}\right)
$$

where

$$
\begin{aligned}
& \dot{Q}_{t o t}=P_{H_{2}}-P_{e l}=I\left(E_{t h} n_{c e l l}-V_{\text {stack }}\right) \\
& \dot{Q}_{\text {cool }}=\rho_{\text {air }} A_{\text {inlet }} v_{a i r} C_{p, a i r}\left(T_{f c}-T_{a m b}\right)
\end{aligned}
$$

Liquid water transport:

$$
\frac{d s}{d t}=\frac{1}{\epsilon_{e f f} d_{C L} \rho_{l} K_{\text {sorp }}}\left(J_{\text {gen }}-J_{\text {evap }}-J_{\text {diff }}\right)
$$

where

$$
\begin{gathered}
J_{\text {gen }}=\frac{I}{2 F A_{\text {geo }}} M_{\mathrm{H}_{2} \mathrm{O}} \\
J_{\text {evap }}=K_{\text {evap }} s \frac{M_{\mathrm{H}_{2} \mathrm{O}}}{R T_{f c} A_{\text {pore }}}\left[p^{0} \exp \left(\frac{-E_{a}}{k_{B} T_{f c}}\right)-p^{v}\right] \\
J_{\text {diff }}=\sigma \cos \theta \sqrt{\epsilon_{\text {eff }} k_{\text {eff }}} \frac{k_{r l}(s) \rho_{l}}{\mu_{l}} \Im(s) \frac{s}{d_{d i f f}} \\
k_{r l}(s)=s^{3} \\
\Im(s)=1.42-4.24 s+3.79 s^{2}
\end{gathered}
$$

The stack voltage is determined by departing from a cross-over-losscorrected theoretical fuel cell potential $E_{0}$ and subtracting activation losses 
as well as ohmic losses. Mass transport losses are neglected due to the opencathode system properties, as described in [12].

$$
V_{\text {stack }}=n_{\text {cell }}\left(E_{0}-\frac{R T_{f c}}{\alpha n F} \ln \left(\frac{I}{A_{\text {geo }} i_{0}{ }^{A E C D}}\right)-I R_{\text {ohm }}\right)
$$

Appendix A.2. State-space model parameters

The parameters $\left(K_{1}\right)$ to $\left(K_{7}\right)$ and the functions $\left(f_{p}\right)$ and $\left(f_{d}\right)$ for the state-space representation are based on the governing equations above.

Parameters:

$$
\begin{aligned}
K_{1} & =\frac{E_{\text {th }} n_{\text {cell }}}{m_{f c} C_{p, f c}} \\
K_{1}^{\prime} & =\frac{1}{m_{f c} C_{p, f c}} \\
K_{2} & =\frac{\rho_{\text {air }} A_{\text {inlet }} C_{p, \text { air }}}{m_{f c} C_{p, f c}} \\
K_{3} & =\frac{M_{H_{2} O}}{2 F A_{\text {geo }} \epsilon_{\text {eff }} d_{C L} \rho_{l} K_{\text {sorp }}} \\
K_{4} & =\frac{K_{\text {evap }} M_{H_{2} O}}{R A_{\text {pore }} \epsilon_{\text {eff }} d_{C L} \rho_{l} K_{\text {sorp }}} \\
K_{5} & =\frac{n_{\text {cell }} R}{\alpha n F} \\
K_{6} & =n_{\text {cell }} R_{\text {ohm }} \\
K_{7} & =n_{\text {cell }} E_{0}
\end{aligned}
$$

Functions:

$$
\begin{aligned}
f_{p}\left(T_{f c}\right) & =\frac{1}{T_{f c}}\left[p^{0} \exp \left(\frac{-E_{a}}{k_{B} T_{f c}}\right)-p^{v}\right] \\
f_{d}(s) & =-\frac{J_{d i f f}}{\epsilon_{e f f} d_{C L} \rho_{l} K_{\text {sorp }}}
\end{aligned}
$$




\begin{tabular}{|c|c|c|}
\hline Parameter & Value & Unit \\
\hline Specific heat capacity of the fuel cell stack, $C_{p, f c}$ & 1260 & $\mathrm{~J} \cdot \mathrm{kg}^{-1} \mathrm{~K}^{-1}$ \\
\hline Fuel cell stack mass, $m_{f c}$ & 0.3 & $\mathrm{~kg}$ \\
\hline Air density @ $25^{\circ} \mathrm{C}, \rho_{\text {air }}$ & 1.184 & $\mathrm{~kg} \cdot \mathrm{m}^{-3}$ \\
\hline Specific heat capacity of air $C_{p, a i r}$ & 1012 & $\mathrm{~J} \cdot \mathrm{kg}^{-1} \cdot \mathrm{K}^{-1}$ \\
\hline Effective cross-section of the cathode housing structure, $A_{\text {inlet }}$ & $8.5 e-3$ & $\mathrm{~J} \cdot \mathrm{kg}^{-1} \cdot \mathrm{K}^{-1}$ \\
\hline Theoretical potential @ $T^{r e f}=25^{\circ} \mathrm{C}$ and $P^{r e f}=1 \mathrm{~atm}, E_{t h}$ & 1.23 & $\mathrm{~V}$ \\
\hline Number of cells in the stack, $n_{\text {cell }}$ & 20 & - \\
\hline Intrinsic exchange current density of $\mathrm{Pt}, i_{0}^{r e f}$ & $5 e-3$ & $A \cdot m^{-2}$ \\
\hline Charge transfer coefficient, $\alpha$ & 0.3 & - \\
\hline Activation barrier for the ORR on $\mathrm{Pt}, \Delta G^{*}$ & 66 & $\mathrm{k} \cdot \mathrm{J} \cdot \mathrm{mol}^{-1}$ \\
\hline Optimal electrode roughness factor, $A^{o p t}$ & 118 & - \\
\hline Optimal reachable liquid water saturation, $s^{o p t}$ & 0.165 & - \\
\hline Geometric catalyst surface area, $A^{g e o}$ & $22.5 e-4$ & $\mathrm{~m}^{2}$ \\
\hline Ohmic stack resistance, $R_{o h m}$ & 0.3 & $\Omega$ \\
\hline Partial pressure of oxygen at the cathode, $p_{\mathrm{O}_{2}}$ & $0.21 \cdot P^{r e f}$ & $\mathrm{~Pa}$ \\
\hline Effective porosity, $\epsilon_{e f f}$ & 0.5 & - \\
\hline Effective Permeability, $K_{e f f}$ & $1 e-14$ & $\mathrm{~m}^{2}$ \\
\hline Liquid water density, $\rho_{l}$ & 970 & $\mathrm{~kg} \cdot \mathrm{m}^{3}$ \\
\hline Liquid water viscosity, $\mu_{l}$ & $3.52 e-4$ & $\mathrm{~Pa} \cdot \mathrm{s}$ \\
\hline Liquid water surface tension, $\sigma$ & 0.0625 & $\mathrm{~N} \cdot \mathrm{m}^{-1}$ \\
\hline Effective contact angle, $\theta$ & 91 & o \\
\hline Effective thickness of diffusion media, $d_{d i f f}$ & $0.4 e-3$ & $\mathrm{~m}$ \\
\hline Catalyst layer volume, $V_{C L}$ & $2.25 e-8$ & $\mathrm{~m}^{3}$ \\
\hline Catalyst layer thickness, $d_{C L}$ & $0.01 e-3$ & $\mathrm{~m}$ \\
\hline Sorption time constant, $K_{\text {sorp }}$ & 360 & - \\
\hline Evaporation time constant, $K_{\text {evap }}$ & $8.6 e 5$ & - \\
\hline Pre-exponential factor, $p^{0}$ & $1.196 e 11$ & $\mathrm{~Pa}$ \\
\hline Activation energy of evaporation, $E_{a}$ & 0.449 & $\mathrm{eV}$ \\
\hline Boltzmann constant, $k_{B}$ & $8.617 e-5$ & $\mathrm{eV} \cdot \mathrm{K}^{-1}$ \\
\hline Cathode ambient pressure, $P_{a m b}$ & $1.013 e 5$ & $\mathrm{~Pa}$ \\
\hline Cathode ambient temperature, $T_{a m b}$ & 298 & $\mathrm{~K}$ \\
\hline Cathode ambient vapor pressure @ $75 \% R H, p^{v}$ & 2380 & $\mathrm{~Pa}$ \\
\hline Pore surface area per unit volume, $A_{\text {pore }}$ & $2 e 7$ & $\mathrm{~m}^{2} \cdot \mathrm{m}^{-3}$ \\
\hline
\end{tabular}

Table A.1: Physical properties and constants 


\section{List of Tables}

A.1 Physical properties and constants . . . . . . . . . . . 30 


\section{List of Figures}

$1 T_{f c}^{*}$ vs $s^{*}$ at equilibrium points. (a) for different levels of $I^{*}$.

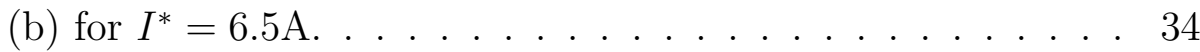

$2 T_{f c}^{*}$ vs $v_{\text {air }}{ }^{*}$ at equilibrium points for differents levels $I^{*}$. . . . 35

3 Eigenvalues of the linearized system in each equilibrium point.

(a) minimum eigenvalue (b) maximum eigenvalue. . . . . . . . 36

4 Equilibrium points stability analysis. Stable equilibrium points are drawn in blue. Unstable equilibrium points are drawn in red. . . . . . . . . . . . . . . . . 37

5 Some trajectories for different initial conditions (around equilibrium points) and values of $v^{*}$ for $I^{*}=6.5 \mathrm{~A}$. . . . . . . . . 38

6 Evolution of $\dot{V}$ in the region of interest, for four different equilibrium points $\left(\mathbf{x}^{*}, \mathbf{u}^{*}\right)$. The figure depicts the value of $\dot{V}$ in color scale, the equi-valued contour curves of $\dot{V}$, the analyzed equilibrium points and the boundary between stable and unstable equilibrium points. (a) $T_{f c}^{*}=315.52 \mathrm{~K}$, $s^{*}=0.108, I^{*}=6.1 \mathrm{~A}$ and $v^{*}=-0.1806$. (b) $T_{f c}^{*}=308.66 \mathrm{~K}$, $s^{*}=0.1169, I^{*}=5 \mathrm{~A}$ and $v^{*}=-0.1444$. (c) $T_{f c}^{*}=304.55 \mathrm{~K}$, $s^{*}=0.1029, I^{*}=3 \mathrm{~A}$ and $v^{*}=-0.08114$. (d) $T_{f c}^{*}=300.55 \mathrm{~K}$, $s^{*}=0.0971, I^{*}=2 \mathrm{~A}$ and $v^{*}=-0.0520 \ldots \ldots$. . . . . . 39

7 Equipower curves for $V_{\text {stack }}^{*}$ vs $T_{f c}^{*} \ldots \ldots$. . . . . . . . 40

$8 \quad$ Stack voltage and current versus $T_{f c}^{*}$ for $70 W$ constant power . 41

9 Closed-loop temperature control system. . . . . . . . . . . . 41 
10 Frequency response of some members of the transfer function family describing the local behavior at relevant equillibrium

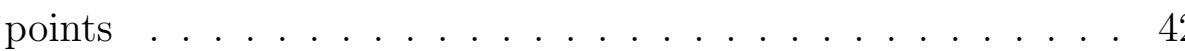

11 Closed-loop step response for some members of the transfer function family describing the local behavior at relevant equillibrium points and the proposed PI controller. (a) System

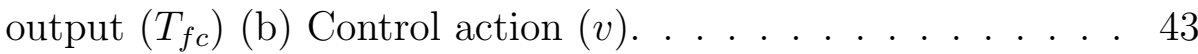

12 Magnitude of the sensitivity function frequency response for some members of the transfer function family with the proposed PI controller, describing the local behavior at relevant equilibrium points. . . . . . . . . . . . . . . 44

13 Temperature profile tracking for constant current using the complete nonlinear model. (a) Temperature $\left(T_{f c}\right)$, (b) Control action $v_{\text {air }}$ ) for $I=4 \mathrm{~A}$. (c) Temperature $\left(T_{f c}\right)$, (d) Control

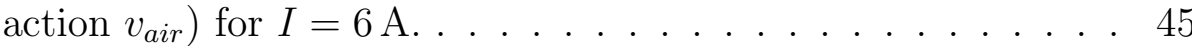




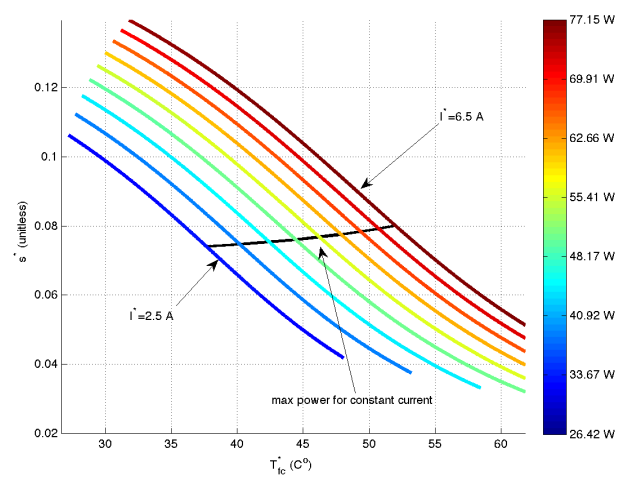

(a)

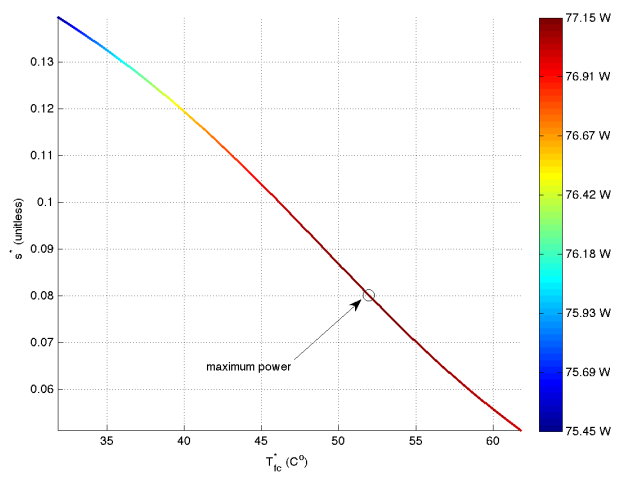

(b)

Figure 1: $T_{f c}^{*}$ vs $s^{*}$ at equilibrium points. (a) for different levels of $I^{*}$. (b) for $I^{*}=6.5 \mathrm{~A}$. 


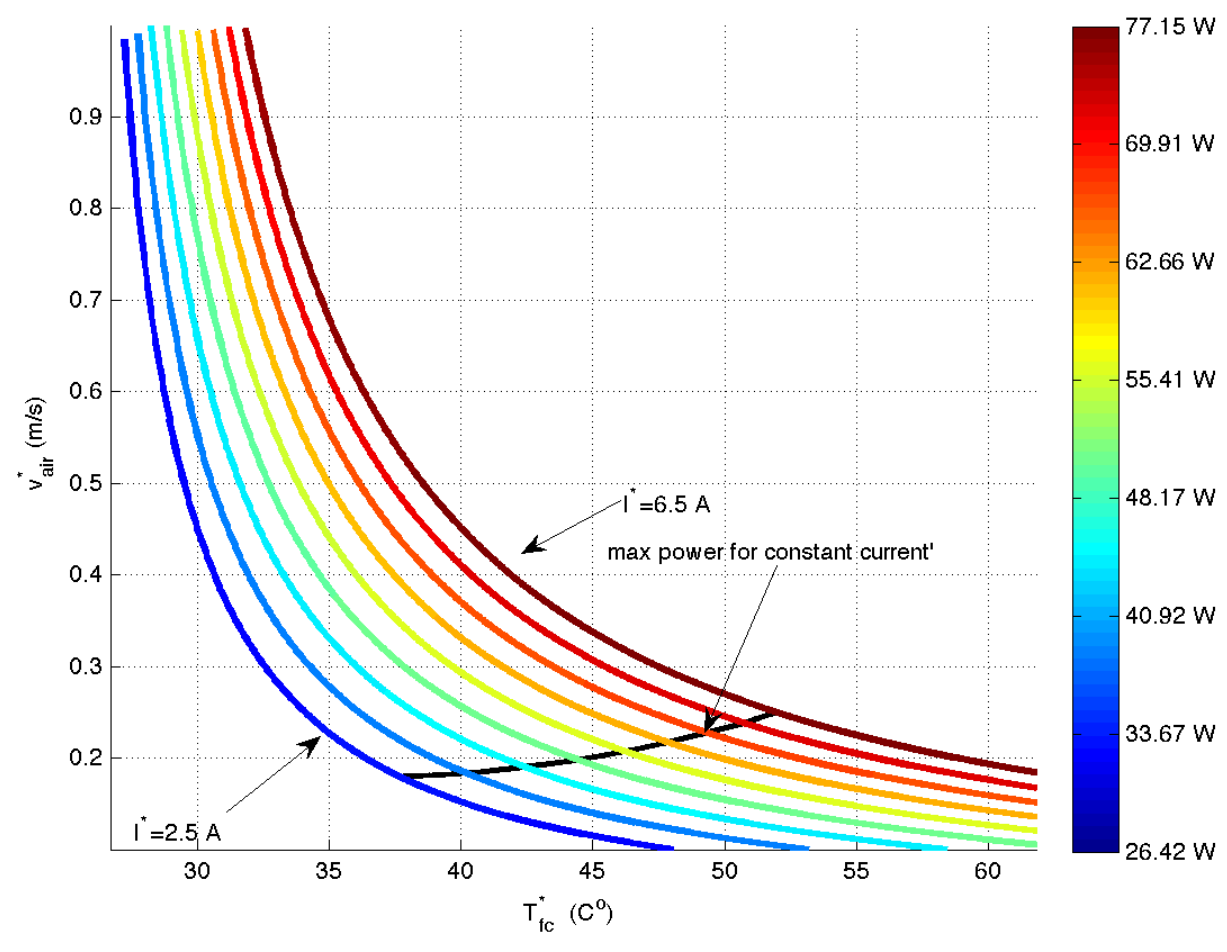

Figure 2: $T_{f c}^{*}$ vs $v_{a i r}{ }^{*}$ at equilibrium points for differents levels $I^{*}$. 


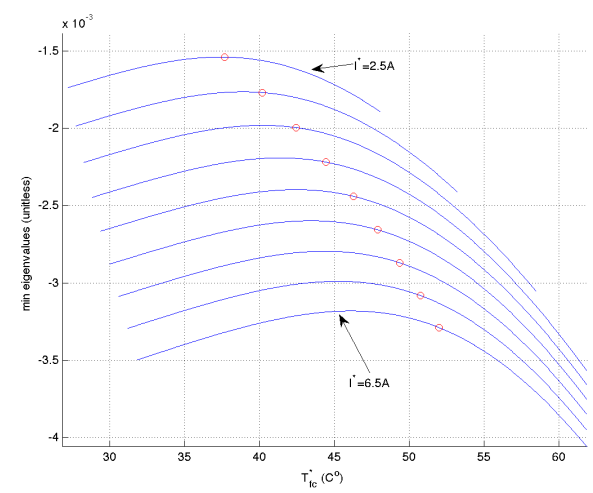

(a)

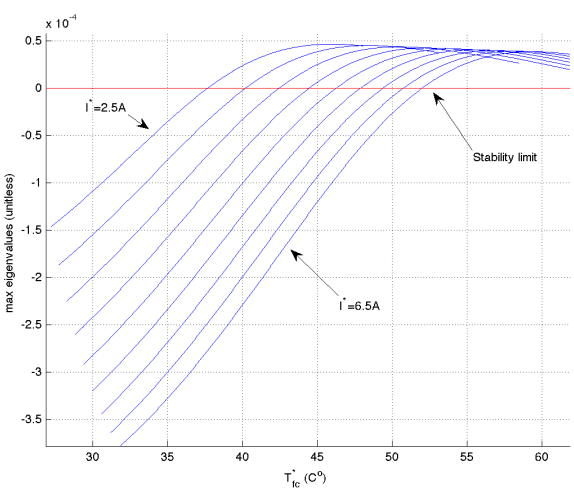

(b)

Figure 3: Eigenvalues of the linearized system in each equilibrium point. (a) minimum eigenvalue (b) maximum eigenvalue. 


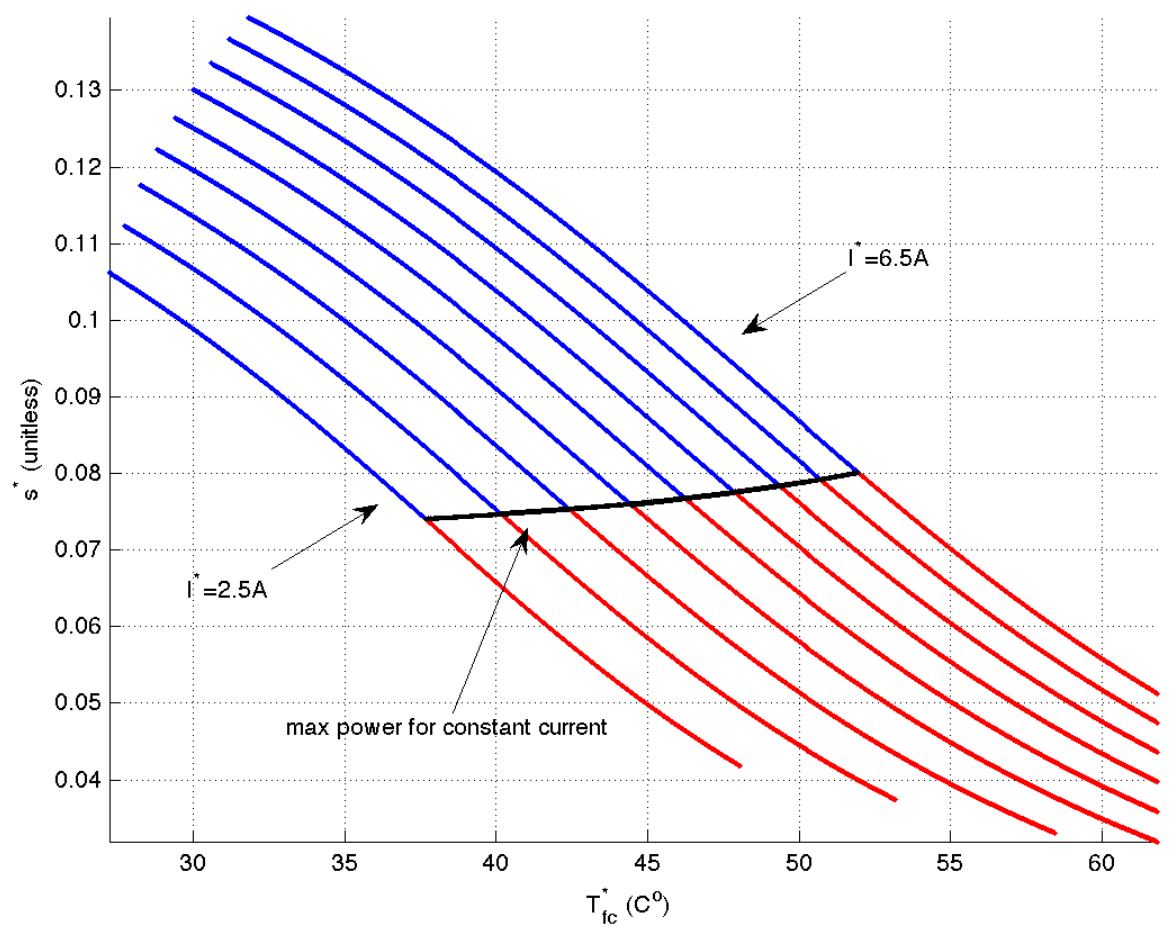

Figure 4: Equilibrium points stability analysis. Stable equilibrium points are drawn in blue. Unstable equilibrium points are drawn in red. 


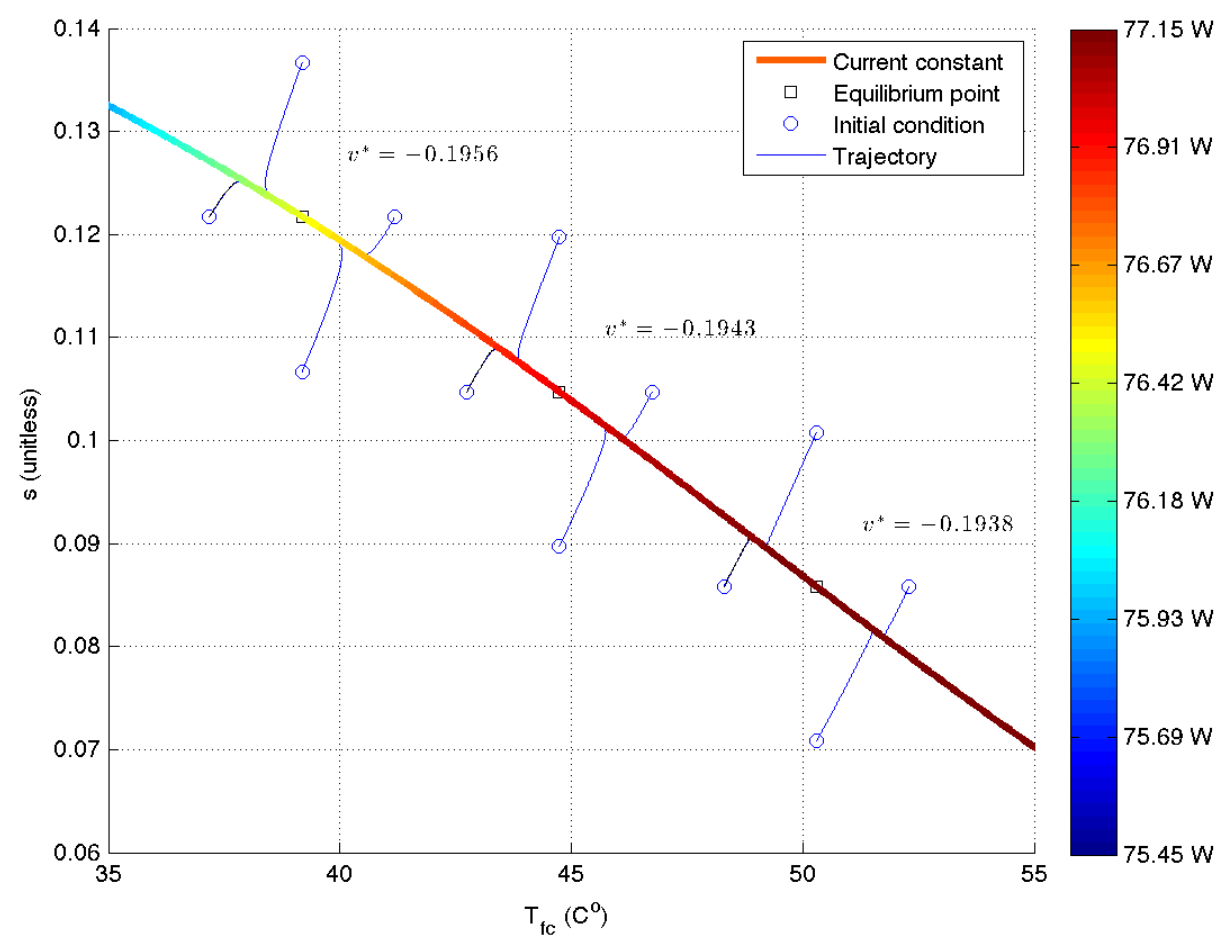

Figure 5: Some trajectories for different initial conditions (around equilibrium points) and values of $v^{*}$ for $I^{*}=6.5 \mathrm{~A}$. 


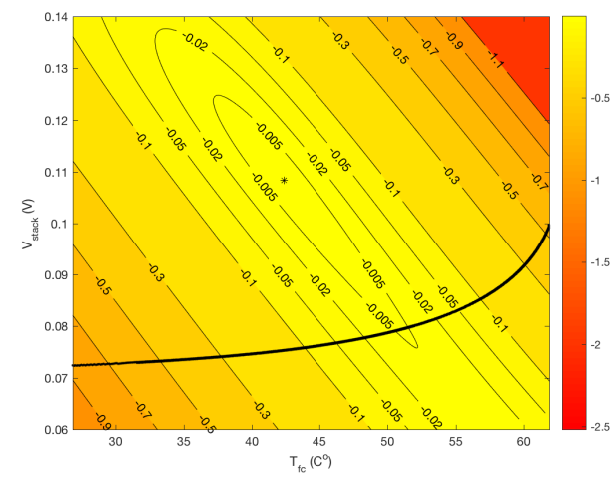

a

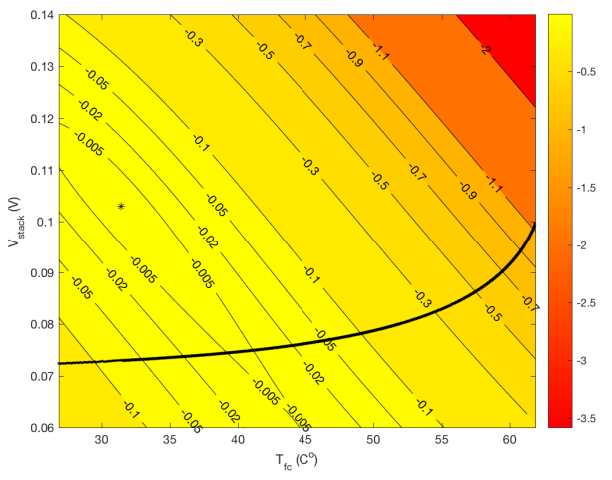

C

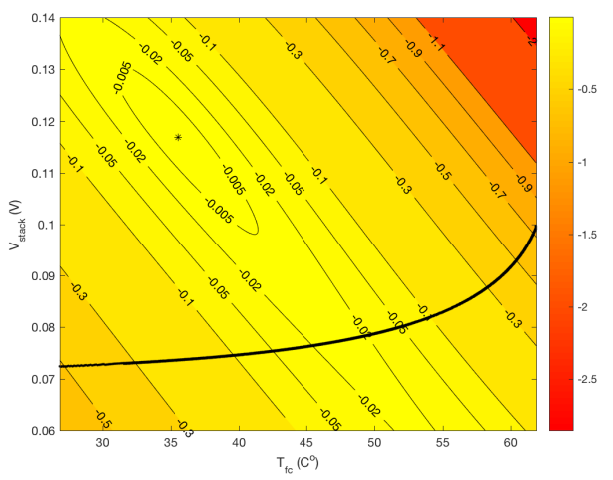

b

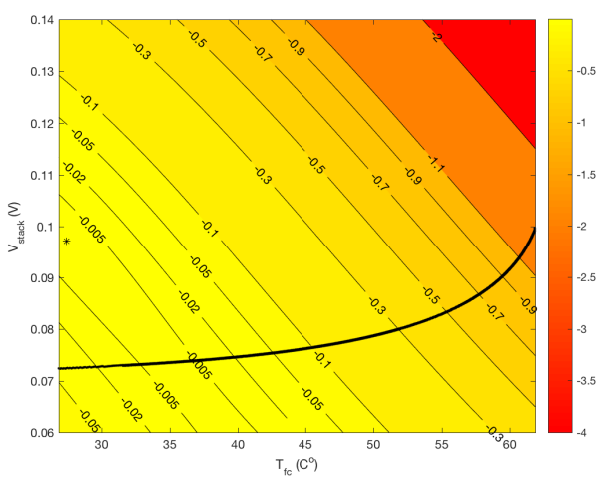

d

Figure 6: Evolution of $\dot{V}$ in the region of interest, for four different equilibrium points $\left(\mathrm{x}^{*}, \mathbf{u}^{*}\right)$. The figure depicts the value of $\dot{V}$ in color scale, the equi-valued contour curves of $\dot{V}$, the analyzed equilibrium points and the boundary between stable and unstable equilibrium points. (a) $T_{f c}^{*}=315.52 \mathrm{~K}, s^{*}=0.108, I^{*}=6.1 \mathrm{~A}$ and $v^{*}=-0.1806$. (b) $T_{f c}^{*}=308.66 \mathrm{~K}, s^{*}=0.1169, I^{*}=5 \mathrm{~A}$ and $v^{*}=-0.1444$. (c) $T_{f c}^{*}=304.55 \mathrm{~K}, s^{*}=0.1029$, $I^{*}=3 \mathrm{~A}$ and $v^{*}=-0.08114$. (d) $T_{f c}^{*}=300.55 \mathrm{~K}, s^{*}=0.0971, I^{*}=2 \mathrm{~A}$ and $v^{*}=-0.0520$. 


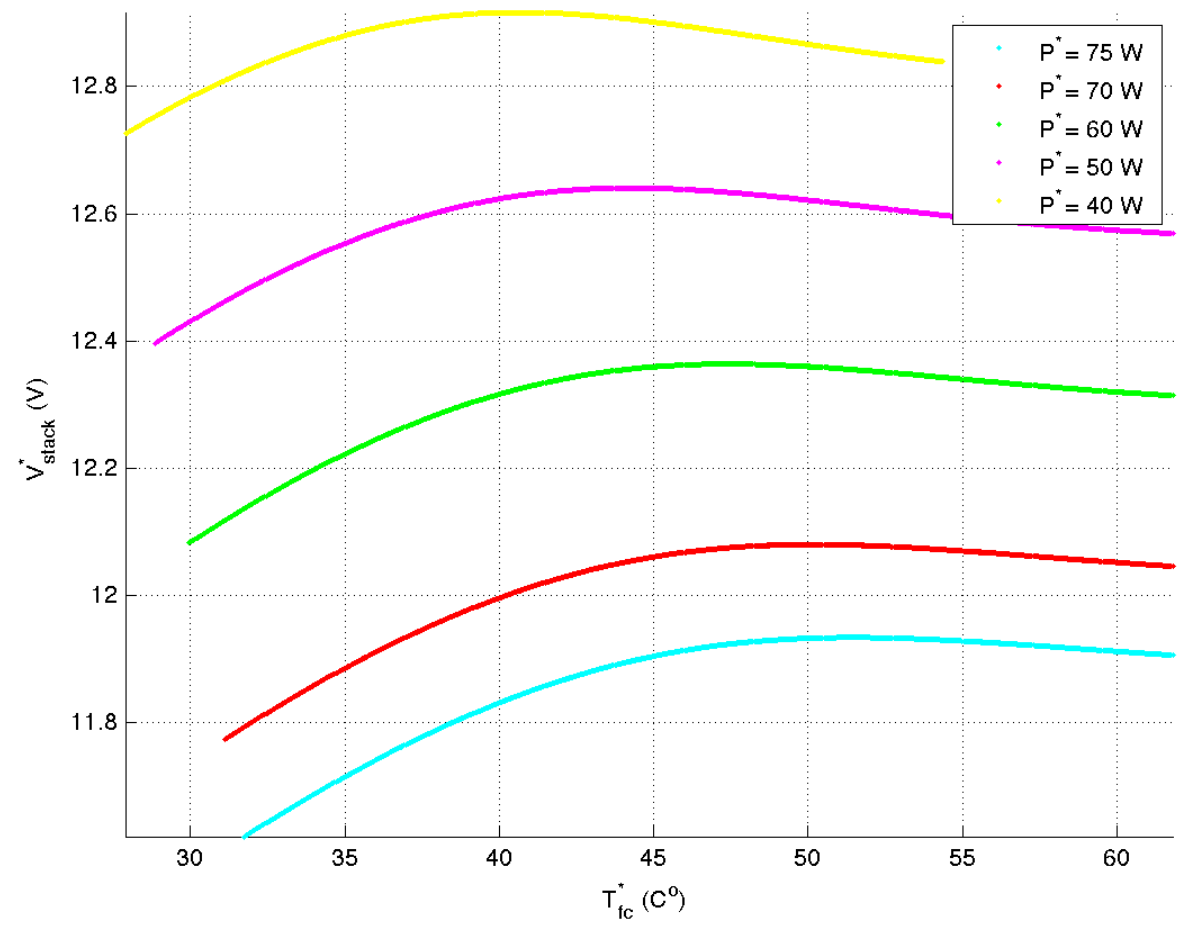

Figure 7: Equipower curves for $V_{\text {stack }}^{*}$ vs $T_{f c}^{*}$ 


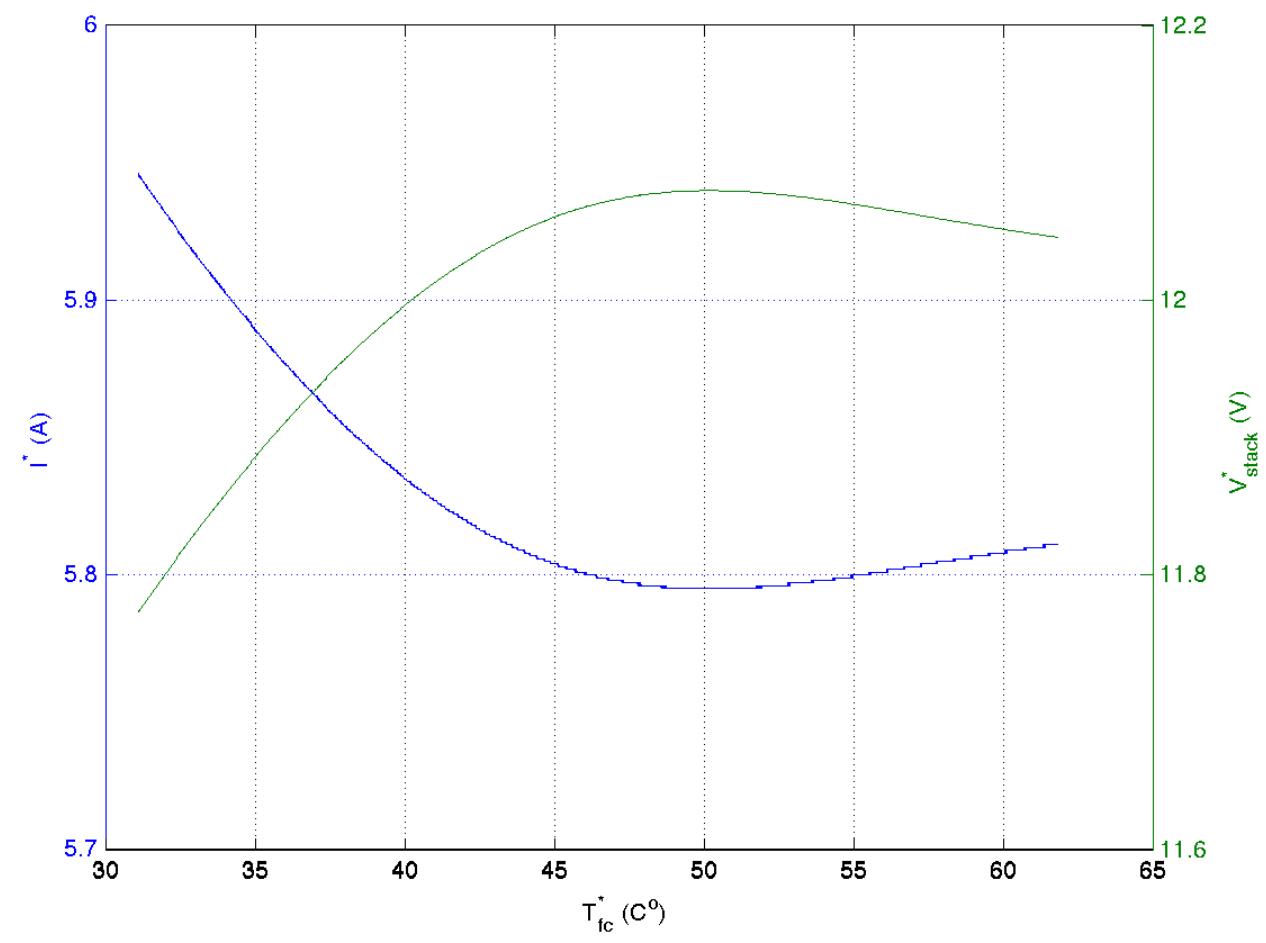

Figure 8: Stack voltage and current versus $T_{f c}^{*}$ for $70 W$ constant power

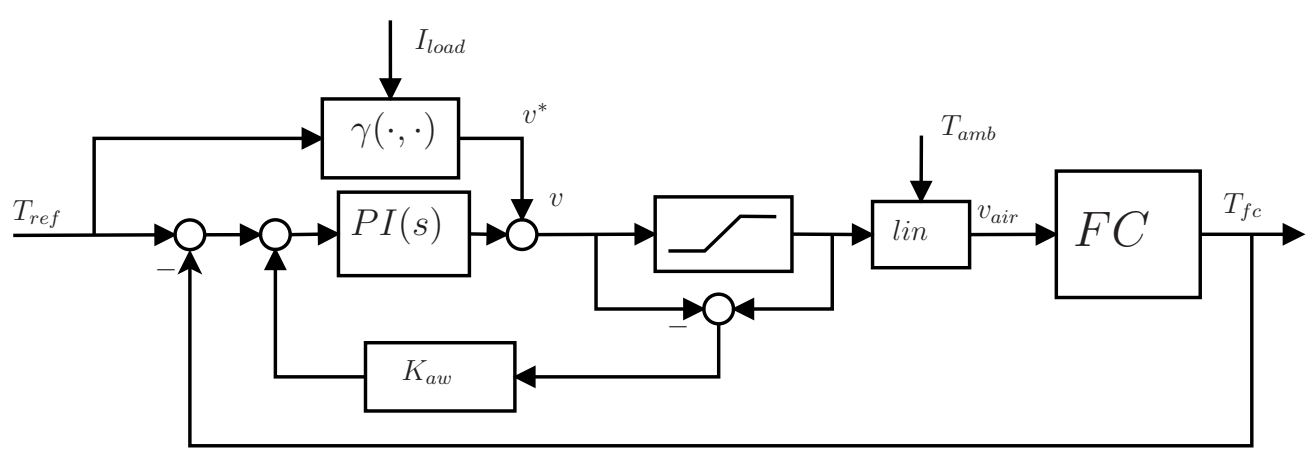

Figure 9: Closed-loop temperature control system. 

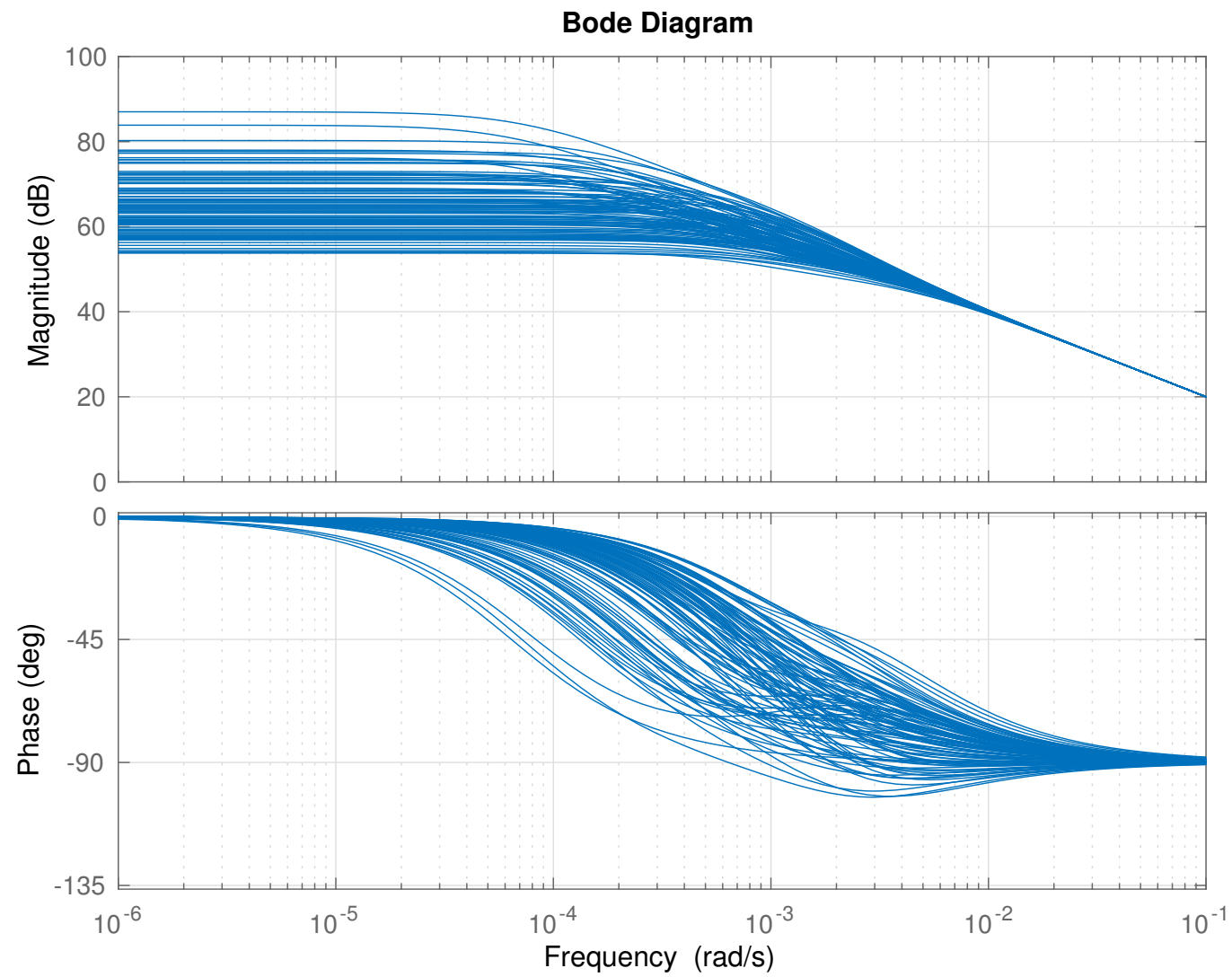

Figure 10: Frequency response of some members of the transfer function family describing the local behavior at relevant equillibrium points 


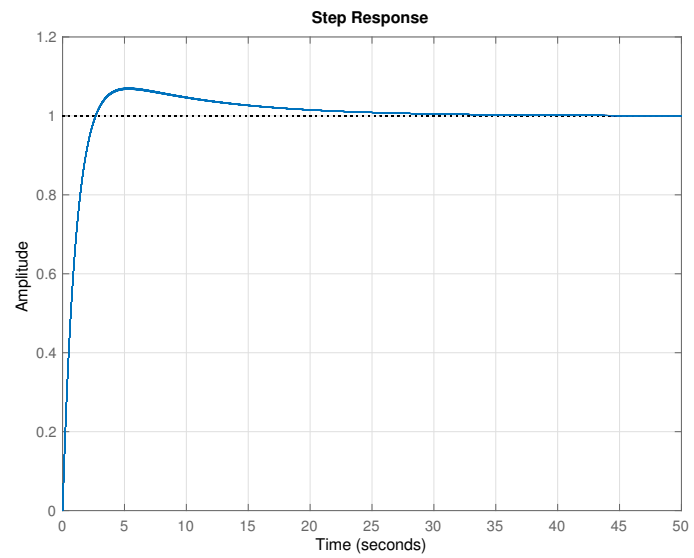

(a)

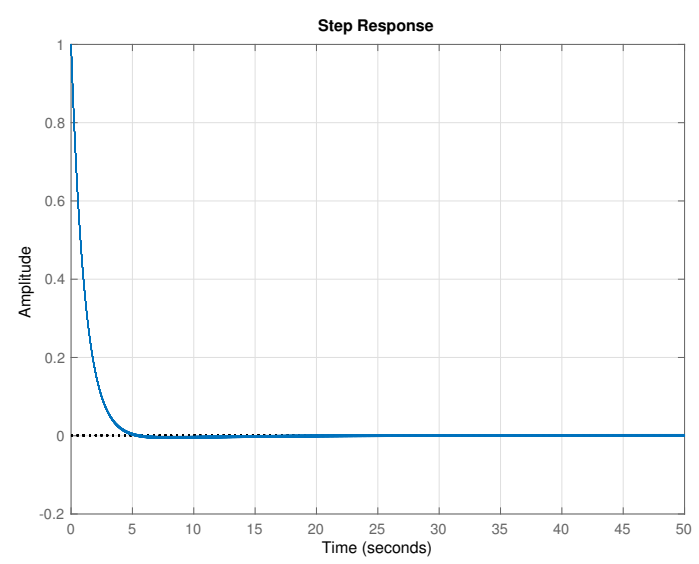

(b)

Figure 11: Closed-loop step response for some members of the transfer function family describing the local behavior at relevant equillibrium points and the proposed PI controller. (a) System output $\left(T_{f c}\right)$ (b) Control action $(v)$. 


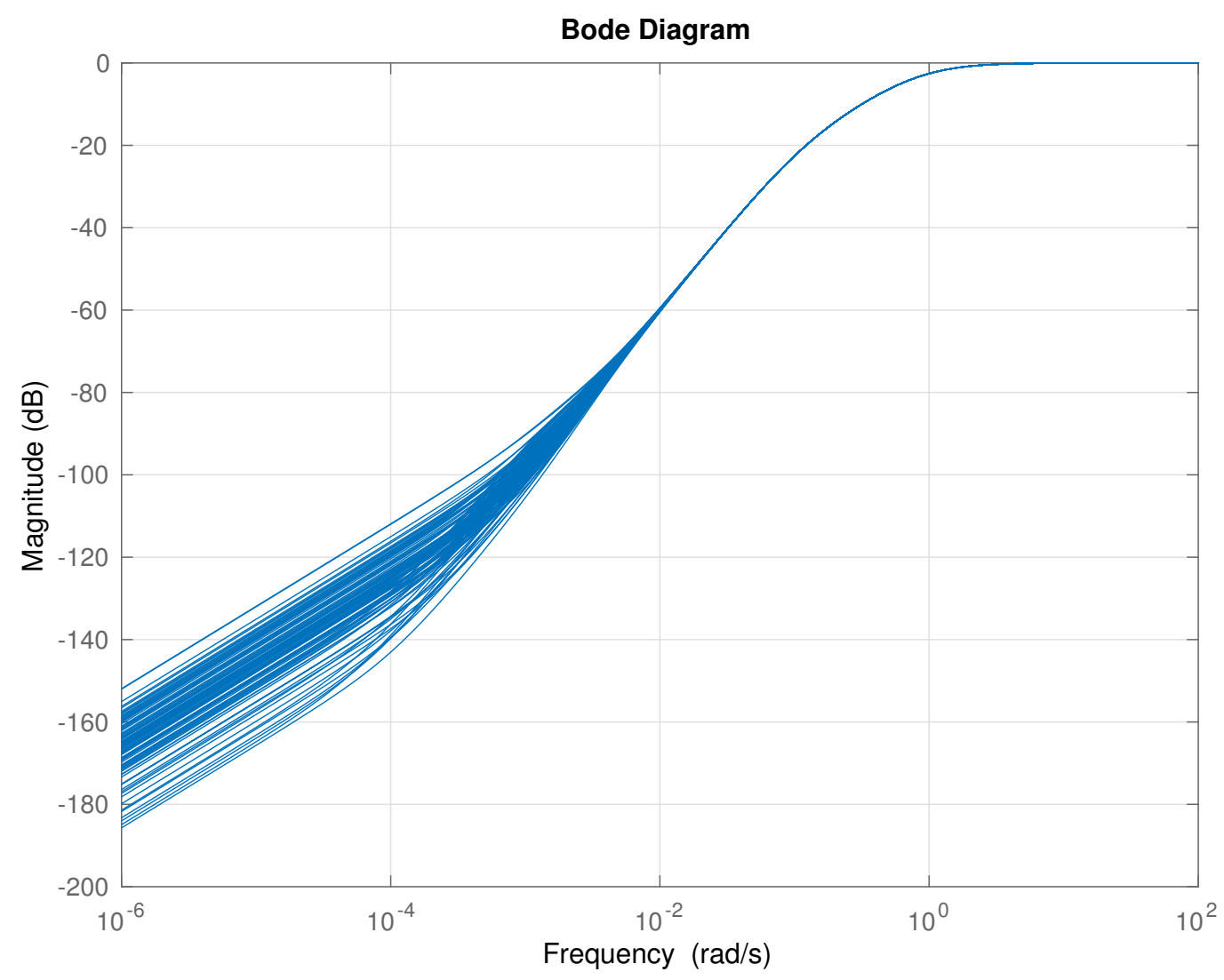

Figure 12: Magnitude of the sensitivity function frequency response for some members of the transfer function family with the proposed PI controller, describing the local behavior at relevant equilibrium points. 


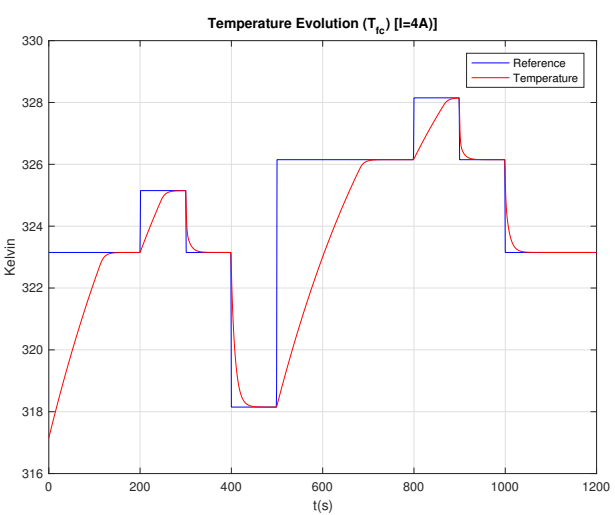

(a)

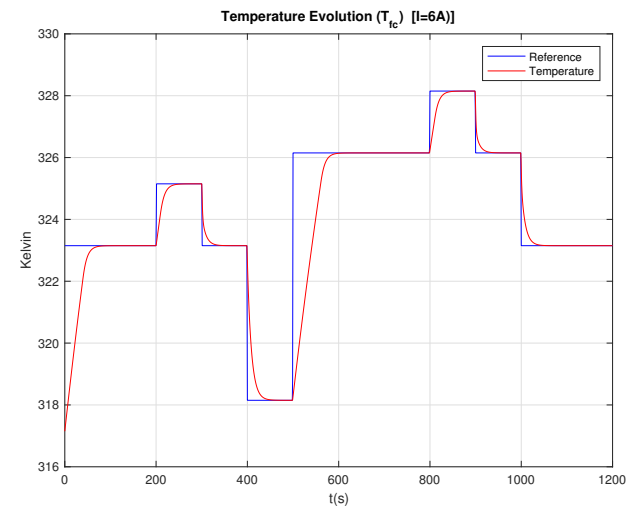

(c)

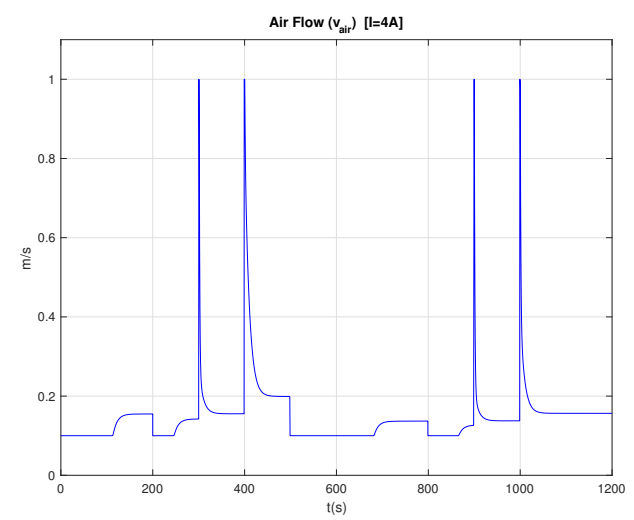

(b)

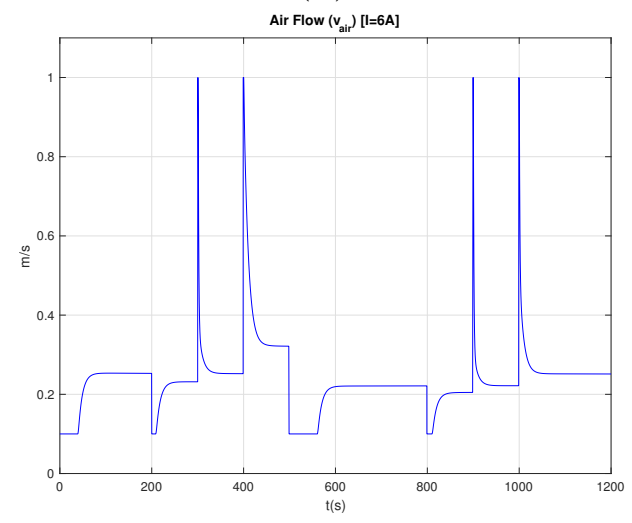

(d)

Figure 13: Temperature profile tracking for constant current using the complete nonlinear model. (a) Temperature $\left(T_{f c}\right)$, (b) Control action $v_{\text {air }}$ ) for $I=4 \mathrm{~A}$. (c) Temperature $\left(T_{f c}\right)$, (d) Control action $\left.v_{\text {air }}\right)$ for $I=6 \mathrm{~A}$. 